\title{
Uso didáctico de las TIC en los colegios rurales agrupados de la Región de Murcia
}

\author{
Didactic Uses of ICT in the Rural Schools in the Region of Murcia
}

\author{
Mํㅗ Pilar Segura García \\ Universidad de Murcia \\ pily.campillos@gmail.com \\ Isabel Mํㅗ Solano Fernández \\ Universidad de Murcia \\ imsolano@um.es \\ Mª del Mar Sánchez Vera \\ Universidad de Murcia \\ mmarsanchez@um.es
}

Recibido: $28 / 09 / 2018$

Aceptado: $3 / 12 / 2018$

Publicado: 21/12/2018

\section{RESUMEN}

Actualmente, vivimos en una sociedad cada vez más digital, donde las Tecnologías de la Información y la Comunicación (TIC) están provocando un impacto en todos los niveles, también en el educativo. La escuela debe asumir el reto de integrar eficazmente las TIC, promoviendo situaciones enriquecidas con tecnologías en las que la metodología constituya el factor clave de los procesos de innovación educativa. La escuela rural debe hacer frente para ello a las desigualdades existentes, con el firme propósito de superarlas. Desde esta perspectiva, el artículo presenta un estudio descriptivo del uso que en las escuelas rurales de la Región de Murcia se hace de las TIC. La metodología escogida es la mixta, y la recogida de información se ha realizado a través de un cuestionario ad hoc construido para la investigación. Con los resultados obtenidos, hemos podido conocer las necesidades en la disponibilidad de recursos, y el uso didáctico que hacen de las TIC los docentes de los colegios rurales agrupados (CRA) en la Región de Murcia, constatando que aún les cuesta dejar atrás metodologías tradicionales para dar paso a la innovación educativa, a través de tecnologías emergentes.

\section{PALABRAS CLAVE}

TIC; escuela rural; innovación educativa; metodología.

\section{ABSTRACT}

Today, we live in an increasingly digital society, where Information and Communication Technologies (ICT) are causing an impact at all ranges, in the educative even. The school must take on the challenge of effectively integrating ICT, promoting situations enriched with technologies in which methodology was the key factor in educational innovation processes. Rural schools must take up this challenge, assuming existing inequalities, but with the firm intention of overcoming them. From this perspective, the article presents a descriptive study of the use of ICT in rural schools in the Region of Murcia. The methodology chosen is mixed, and the collection of information has been carried out through an ad hoc questionnaire constructed for research. From the analysis of data, we have been able to know the needs in the availability of resources, 
and the didactic use that the teachers of the rural schools grouped together in the Region of Murcia make of the ICT, confirming that it is still difficult for them to leave behind traditional methodologies to give way to the educational innovation, through emerging technologies.

\section{KEYWORDS}

ITC; rural school; educational innovation; methodology.

\section{CITA RECOMENDADA}

Segura, M.P., Solano, I.M. y Sánchez, M.M. (2018). Uso didáctico de las TIC en los colegios rurales agrupados de la Región de Murcia. RIITE. Revista Interuniversitaria de Investigación en Tecnología Educativa, 5, 102-115. Doi: http://dx.doi.org/10.6018/riite/2018/343771

\section{Principales aportaciones del artículo y futuras líneas de investigación:}

- La investigación desarrollada, ha permitido conocer la situación real que viven los colegios rurales agrupados de la Región de Murcia en cuanto a TIC.

- Como futuras líneas de investigación, podrían llevarse a cabo estudios que analizasen más ampliamente este tema consiguiendo una muestra mayor, y planteando un estudio más profundo en cuanto a dotación tecnológica se refiere, ya que este aspecto ha sido tratado de forma leve.

- Sería conveniente realizar estudios cualitativos, que pudieran analizar la percepción que tiene el profesorado en cuanto a la desigualdad o diferencia que encuentran entre este tipo de centros y otros urbanos, y en el sentido que se produce.

\section{INTRODUCCIÓN}

Las tecnologías suponen, hoy en día, un aspecto fundamental en nuestra vida diaria. Nos encontramos inmersos en una sociedad en la que la tecnología de la información y la comunicación está presente en todos los ámbitos, ofreciéndonos la posibilidad de relacionarnos de forma eficaz y sencilla con el medio que nos rodea, además de favorecer un desarrollo acorde con las necesidades de la sociedad actual. Por este motivo, cobra especial importancia que el ámbito educativo, desde bien temprano, prepare a personas competentes para hacer frente y adaptarse a esta sociedad de la información. Así lo establece el Marco Europeo de competencia digital para el ciudadano DIGComp 2.0 (Vuorikari, Punie, Carretero, Van Den Brande, 2016), y orientado al ámbito docente, en el que nos centramos en este trabajo, el Marco Común de la Competencia Digital Docente (INTEF, 2017).

Debido a los cambios que está sufriendo nuestra sociedad actual, no cabe duda de la necesidad de que los ciudadanos requieran de sistemas y de escuelas que incorporen los recursos tecnológicos a los procesos de enseñanza y aprendizaje. La integración de las TIC en el ámbito educativo es actualmente un tema de capital importancia desde muchas perspectivas y en todos los niveles educativos. Por esto, el esfuerzo de dotar de dichas tecnologías a las escuelas, de tal forma que los estudiantes dispongan y sepan utilizarlas para poder hacer frente a lo que demanda la sociedad (Bustos y Román, 2016).

Es evidente que, ante esta revolución, la escuela no puede permanecer impasible, y menos la escuela rural, teniendo la obligación de adaptarse a esta realidad. Pero, dentro del ámbito educativo, siempre han estado presentes las desigualdades. Hasta hace bien poco, los centros situados en contextos rurales se encontraban en una situación de verdadera desventaja, al estar alejadas del medio urbano, y de las oportunidades que ofrecen las grandes ciudades. El medio rural sufría una situación de aislamientos tecnológico, con infraestructuras deficitarias que no contribuían a un progreso y desarrollo de la población (Del Moral y Villalustre, 2007). 
A lo largo del tiempo, la escuela rural en España ha sido la gran olvidada, tanto por los padres, alumnos, y profesores, como por la administración pública. Las dificultades económicas que sufría este medio en nuestro país durante el siglo XIX y gran parte del siglo XX, provocaron que la gente estuviera más preocupada en no morir de hambre, que en la educación de los más pequeños (Bustos, 2006; Corchón, 2005; Hinojo, Raso e Hinojo, 2010; Raso, 2012).

Sin embargo, ahora, ya en el siglo XXI, a partir del punto de vista socioeconómico se han producido muchos cambios, aunque la escuela sigua siendo aún una marginada por parte de la comunidad educativa.

El contexto rural se transforma y sus formas tradicionales están desapareciendo. Se está produciendo un proceso de cambio acelerado, la cultura rural se moderniza progresivamente gracias a su introducción en el sistema económico global. Además, debido a los medios de comunicación, las relaciones entre el contexto rural y el urbano han aumentado, dando lugar a eliminar ese aislamiento característico. El desarrollo de dichos medios transmite a ambos, una formación mediática que los uniforma en cuanto a hábitos, ideas, valores, entre otras (Bustos, 2006).

Las escuelas se han convertido en grandes y modernas instituciones, nada tienen que ver con las que acostumbrábamos, "aquellas casuchas raídas de polvo y telarañas que amenazaban día a día con caerse a pedazos" (Raso, hinojo y Solá, 2015, p.140). Las actuales, ya hacen uso de las TIC, y se han unido a la Sociedad del Conocimiento, además de que ofrecen a los niños la educación en un medio saludable y libre de aires contaminados (Raso, hinojo y Solá, 2015).

Teniendo en cuenta todo esto, se considera que las TIC juegan un papel fundamental para reducir estas diferencias. Con la llegada de la Sociedad de la Información, uno de los temas sobre el que estamos todos de acuerdo, es en la gran importancia que representa el uso de las nuevas tecnologías para las generaciones de hoy en día, de forma que éstas sean para los alumnos algo tan habitual como lo es escribir o leer (Cruz, Torres, López y Ballesteros, 2004).

Las TIC son consideradas como poderosas herramientas, canales y soportes que almacenan, sintetizan, procesan, recuperan y transmiten todo tipo de información de distintas formas, para satisfacer las necesidades de nuestra actual sociedad. Suponen, un gran cambio para la educación, y abren un mundo de posibilidades para la enseñanza en la escuela rural, ya que transforman las relaciones interpersonales, el modo de producir conocimiento, y de difundir información rompiendo barreras geográficas. Por todo esto, las instituciones educativas están obligadas a adaptarse a la nueva situación, para conocer y manejar el lenguaje digital y las nuevas maneras de comunicarse (Alcántara, 2009; Cruz et al., 2004).

Por consiguiente, los maestros no pueden impartir la educación al margen de los cambios que se llevan a cabo en nuestra sociedad, ha sido necesario realizar un gran esfuerzo para poder llevar las nuevas tecnologías a la escuela rural. Dicho proceso produce multitud de cambios en los centros, uno de ellos es la necesidad de formación permanente que debe tener el profesorado, ya que, es el encargado de acceder a la información, procesarla, transformarla y transmitirla a sus alumnos, para estar actualizados y preparados en su labor, mejorando e innovando en el proceso de enseñanza-aprendizaje (Cabero, 2015).

Las zonas menos favorecidas, como es el caso del medio rural, han sido uno de los objetivos de muchas acciones llevadas a cabo por parte del gobierno, de forma que puedan tener acceso a nuevas oportunidades, algunas de ellas relacionadas con la introducción de las TIC para intentar disminuir las desventajas características, tanto de este tipo de centros particularmente, como en el contexto económico y sociocultural, de manera más general.

Dentro del marco educativo, según Del Moral, Villalustre y Neira (2014), en las últimas décadas, también se han llevado a cabo proyectos institucionales para impulsar las TIC, como Aldea Digital y Programa Escuela 2.0, y más concretamente en la zona de Andalucía han tenido lugar El Plan Alhambra, el Plan andaluz de introducción a las nuevas tecnologías de la imagen y la comunicación (P.A.I.N.T.I.C.), los Programas Redaula o Aul@bus, entre otros (Recio, 2016). Todos ellos destinados a abastecer con programas educativos y recursos tecnológicos a las escuelas rurales, con el objetivo de disminuir la brecha digital que se había creado entre entornos rurales y urbanos. 
Al hilo de lo expuesto anteriormente, se han producido cambios tanto en los procesos de enseñanza, como en las prácticas llevadas a cabo como consecuencia de la digitalización de las escuelas, se ha producido una transformación que ha afectado tanto a los nuevos roles de los docentes, como a las estrategias puestas en práctica. Sin embargo, una verdadera innovación no se debe solamente a la incorporación de las TIC en las aulas, sino en el papel que desempeñan los maestros a la hora de integrarlas adecuadamente para favorecer un aprendizaje (Del Moral, Villalustre y Neira, 2014). Es por ello, la importancia de una formación permanente ante las nuevas tecnologías que emergen hoy en día.

Una vez hecha la revisión teórica, llegamos al problema en torno al cual va a girar toda la investigación, este hace referencia a la integración de las TIC en la escuela rural.

Por ello, para abordar esta problemática se ha considerado oportuno analizar cuál es la disponibilidad y el uso didáctico que hacen de las TIC los docentes de Educación Infantil y Primaria en los Colegios Rurales Agrupados de la Región de Murcia, averiguando además la formación que presentan en relación con este tema, y el grado de enseñanza innovadora a través del empleo de pedagogías y tecnologías emergentes.

\section{MÉTODO}

\subsection{Objetivos}

Para el desarrollo de este estudio, se han planteado una serie de objetivos de investigación desglosados en generales y específicos, a través de los cuales ha girado todo el proceso de recogida de datos, son los siguientes:

Objetivo general 1: Conocer el uso que los docentes de los Colegios Rurales Agrupados de Educación Infantil y Primaria de la Región de Murcia hacen de las TIC.

- Conocer los recursos TIC y la frecuencia de uso por los docentes en las aulas de Educación Infantil y Primaria de los CRA de la Región de Murcia.

- Conocer la formación que han recibido y que reciben los docentes de Educación Infantil y Primaria de CRA de Región de Murcia respecto a Los recursos TIC.

Objetivo general 2: Analizar las estrategias metodológicas enriquecidas con TIC llevadas a cabo por los docentes de Educación Infantil y Primaria de los Colegios Rurales Agrupados en la Región de Murcia.

- Conocer el uso que los docentes de Educación Infantil y Primaria realizan de las tecnologías emergentes en los CRA de la Región de Murcia.

- Identificar las estrategias metodológicas enriquecidas con TIC que han puesto en práctica los docentes de Educación Infantil y Primaria en los CRA de la Región de Murcia.

\subsection{Diseño de la investigación}

De acuerdo con la naturaleza de los datos que se han analizado, por un lado, el uso que hacen de las TIC los docentes de Educación Infantil y Primaria en los Colegios Rurales Agrupados de la Región de Murcia, y por otro lado, las metodologías enriquecidas con TIC que ponen en práctica, se ha llevado a cabo una investigación con un enfoque metodológico no experimental, de corte descriptivo y desde una metodología mixta, pues aunque se utiliza un cuestionario y un posterior análisis de los datos tras la conversión de las categorías en valores numéricos, la mayoría de las variables son nominales y ordinales, por lo que la naturaleza de la información es fundamentalmente cualitativa, y el análisis de los datos descriptivo mediante el análisis de frecuencias y porcentajes (Moscoso, 2017). De esta forma, lo que se pretende es examinar y describir por una parte, el nivel de competencia digital, a través de conocer los recursos TIC de los que disponen, la formación que han recibido y reciben actualmente los docentes, además de la frecuencia y el uso que hacen de ellos en los CRA de la Región de Murcia, y por otra parte, conocer el grado de enseñanza innovadora que se produce, a través de las tecnologías emergentes que conocen y utilizan, y las estrategias metodológicas enriquecidas con TIC que ponen en práctica. 


\subsection{Participantes}

La población y la muestra invitada para la realización del estudio, corresponde a todo el profesorado de Educación Infantil y Primaria que imparte docencia en los ocho Colegios Rurales Agrupados que se encuentran en la Región de Murcia. En cuanto a la muestra productora de datos, un total de 54 docentes han sido los participantes en dicha investigación, presentando las siguientes características: la mayoría está formada por mujeres (69\%), 38 años es la media de edad que presentan (con una desviación típica de 8,125 ), un $57 \%$ pertenece al grupo de la diplomatura en el nivel máximo de estudios alcanzados, la gran mayoría (81\%) son de la especialidad de Educación Primaria, y 12 años es la media sobre la experiencia docente (con una desviación típica de 7,374).

\subsection{Instrumento de recogida de datos}

Se ha diseñado un cuestionario ad hoc construido para la investigación. Se diseñó, para su administración en red a través de Google Forms. El cuestionario está organizado en una serie de bloques, y el número total de ítems es de 48 , combinando tanto preguntas de carácter abierto como cerrado. Son los siguientes:

- Bloque I. Preguntas básicas. Donde se recogen datos relativos al perfil de los participantes, como: sexo, edad, nivel máximo de estudios alcanzado, especialidad y años de experiencia docente.

- Bloque II. Recursos disponibles. En este bloque encontramos una serie de preguntas sobre la disponibilidad y la frecuencia de uso de determinados recursos, y sobre cómo es la conexión a la red en su centro.

- Bloque III. Formación docente. Nos permite obtener datos sobre la formación que han recibido tanto en la universidad, como en contextos no formales, además de la formación continua que reciben y la importancia que le dan.

- Bloque IV. Tecnologías y metodologías emergentes. Compuesto por preguntas que nos indican el uso de tecnologías emergentes, la frecuencia de uso de determinadas estrategias metodológicas, además de dos preguntas de carácter abierto donde los participantes tienen la posibilidad de escribir otras estrategias, y el cambio metodológico que han supuesto las TIC en sus clases.

Dicho instrumento ha sido validado por expertos en la materia usando para ello una ficha de validación, en la que se indicaban aspectos como la pertinencia, univocidad, la importancia, y las observaciones que se consideraran necesarias para mejorar. Una vez validado y modificado el cuestionario, se ha procedido a su distribución entre los docentes de los ocho colegios rurales agrupados de la Región de Murcia. En un primer momento se envió el enlace por correo electrónico a cada uno de los centros, sin embargo, debido a la escasa participación se procedió a difundirlo vía WhatsApp, lo cual les resultaría más cómodo y sencillo de realizar.

\subsection{Análisis de datos}

El tratamiento y análisis de datos se ha realizado conforme al carácter descriptivo de nuestra metodología, utilizando técnicas como el sistema categórico para la parte cualitativa, y como el programa SPSS para la estadística descriptiva de la parte cuantitativa.

El tratamiento estadístico se ha realizado utilizando el programa informático SPSS Statistics, el cual, nos permite sacar frecuencias y porcentajes, a través de tablas y gráficos de las variables del estudio, de manera que el análisis de los datos y las representaciones nos facilitan en gran medida la tarea de su interpretación y análisis exploratorio.

Por otro lado, atendiendo a que tenemos una muestra pequeña, y además, solo hay dos preguntas abiertas en el cuestionario, se ha determinado hacer un análisis de contenido manual, a través de la reducción de los datos. Para su análisis, se ha procedido a leer los comentarios y establecer un sistema de categorías, para posteriormente atribuir códigos. En función de las categorías, se ha analizado la frecuencia con la que aparecen. 
Para finalizar, se redactaron las conclusiones a las que se había llegado en base al análisis de los datos obtenidos.

\section{RESULTADOS}

\subsection{Recursos tecnológicos}

\subsubsection{Recursos tecnológicos disponibles en el aula}

Como vemos en los resultados que muestra la Figura 1, la gran mayoría de los recursos tecnológicos presentados, se encuentran disponibles para su uso en las aulas. Sin embargo, es cierto que tres de ellos, las tablets, los ordenadores portátiles y los smartphone, son difíciles de encontrar en los CRA de la Región de Murcia. De este modo, un 36\% de los docentes no disponen de Tablets para impartir sus clases, un $18 \%$ no dispone tampoco de ordenadores portátiles, y en cuanto a los Smartphone un $17 \%$ de los docentes no cuenta con este recurso para su aula. Además, podemos señalar que los ordenadores fijos son los más comunes, a la vez que los proyectores y la Pizarra Digital Interactiva. Solamente un 3\% de ellos no dispone de ordenador fijo, un $10 \%$ no cuenta con proyectores, y en cuanto a la PDI es un $16 \%$ de docentes el que no tiene a su disposición este recurso.

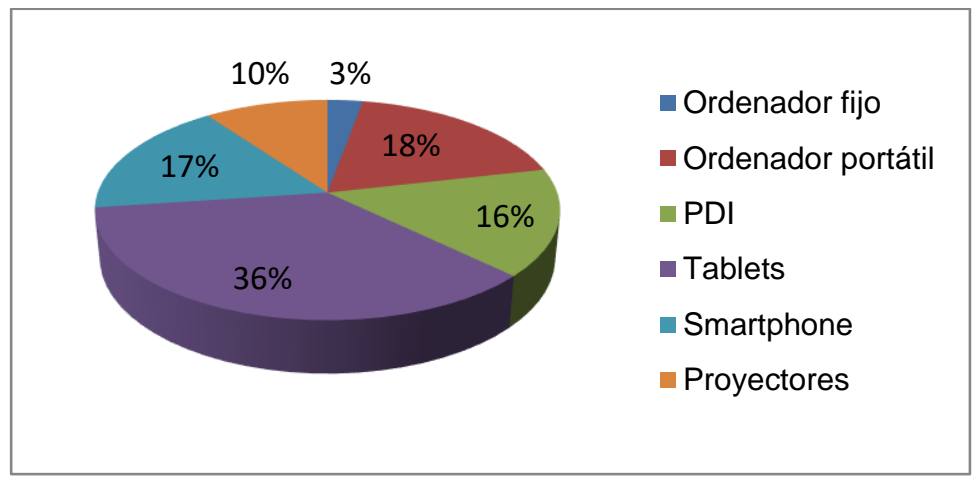

Figura 1. Recursos menos disponibles

\subsubsection{Frecuencia de uso de los recursos disponibles}

Los resultados que se muestran en la Figura 2, nos hacen comprobar que los recursos más utilizados por los docentes son, en mayor medida el ordenador fijo con un porcentaje casi del $50 \%$ que indica que lo suelen utilizar siempre, y seguidamente con un porcentaje del $51,8 \%$ de los docentes, indica que la PDI es utilizada con una frecuencia de a menudo y siempre.

Por el lado contrario, los recursos menos utilizados con la frecuencia de nunca y alguna vez son las tablets con un porcentaje de $35,2 \%$ y el Smartphone con un $61,1 \%$. En cuanto a los proyectores, suelen ser utilizados alguna vez con un $27,8 \%$, y con un $38,9 \%$ también suelen utilizarse alguna vez los ordenadores portátiles. 


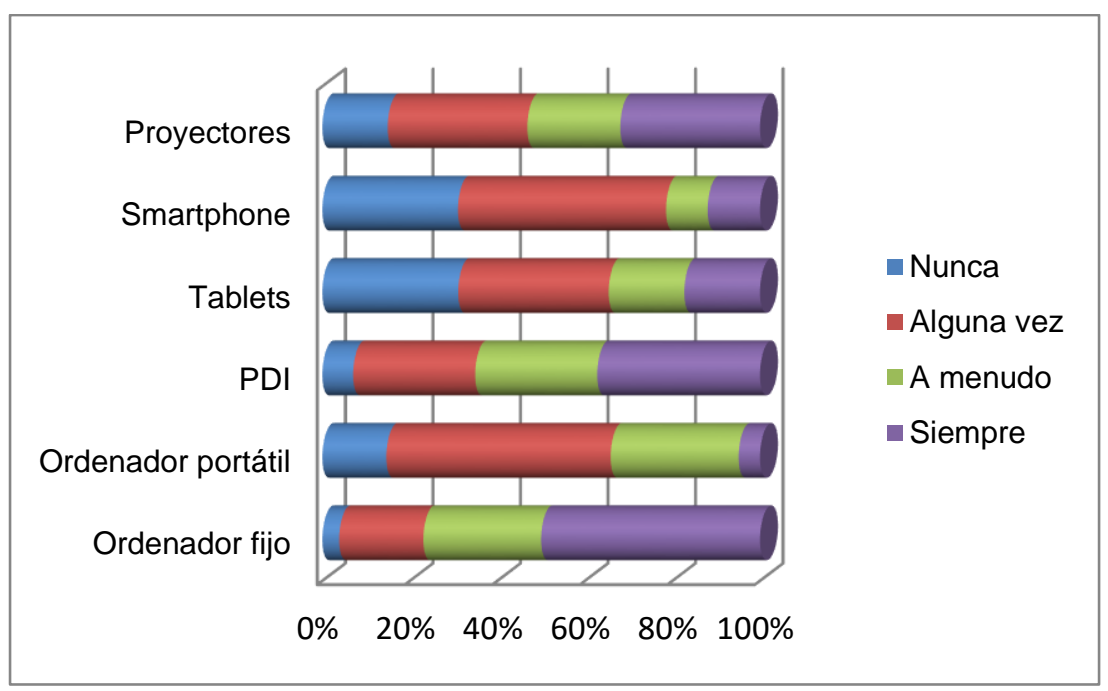

Figura 2. Frecuencia del uso de recursos

\subsubsection{Conexión a la red}

Como podemos observar en la gráfica sobre la conexión a Internet (Figura 3), de los docentes que participan en el estudio, un 64\% afirman tener una conexión a Internet de banda ancha, el $23 \%$ no tienen banda ancha, mientras que existe un $13 \%$ que no conoce cuál es la conexión en su centro.

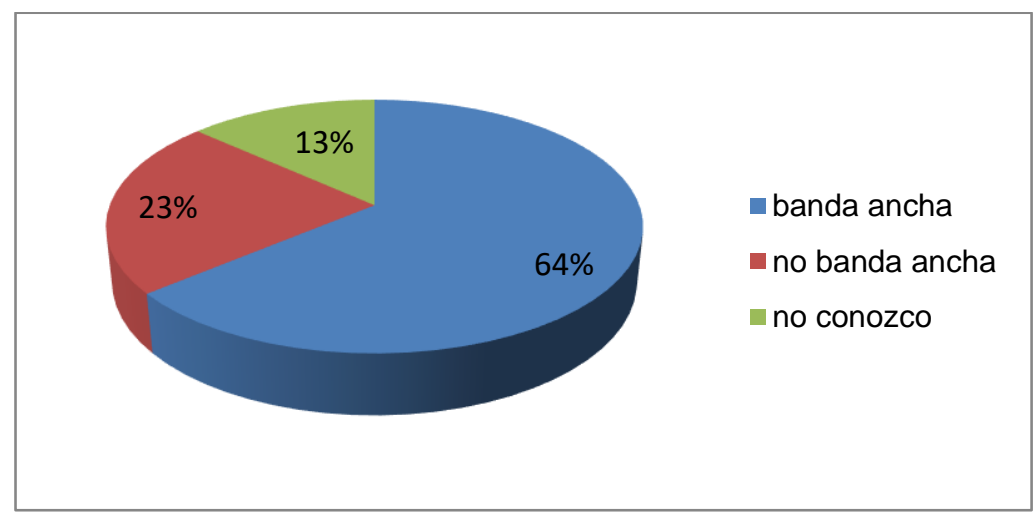

Figura 3. Conexión a la red

\subsection{Formación docente}

Atendiendo a los resultados obtenidos, podemos comprobar (Figura 4), que solamente un $29,6 \%$ de los docentes recibieron formación específica sobre nuevas tecnologías en la universidad, mientras que, es más común entre ellos, con un $57,4 \%$, que lo hicieran en otros contextos como es el caso de academias, seminarios o cursos, o que aprendieran por cuenta propia a través de sus propios medios, $59,3 \%$.

Un 50\% afirma haber recibido formación durante su labor docente, y un $42,6 \%$ ha participado en proyectos de incorporación de TIC en el centro o aula.

Por otro lado, creen en la necesidad de una formación inicial en la materia con un 57,4\%, y además un $61,1 \%$ de ellos piensan que para ser un profesor competente debería ser imprescindible una formación continua. 
Sin embargo, es relevante que solo un $18,5 \%$ de los docentes tenga previsto realizar alguna actividad de formación continua, o que igualmente con un $18,5 \%$, en sus centros se desarrollen planes de formación para el profesorado.

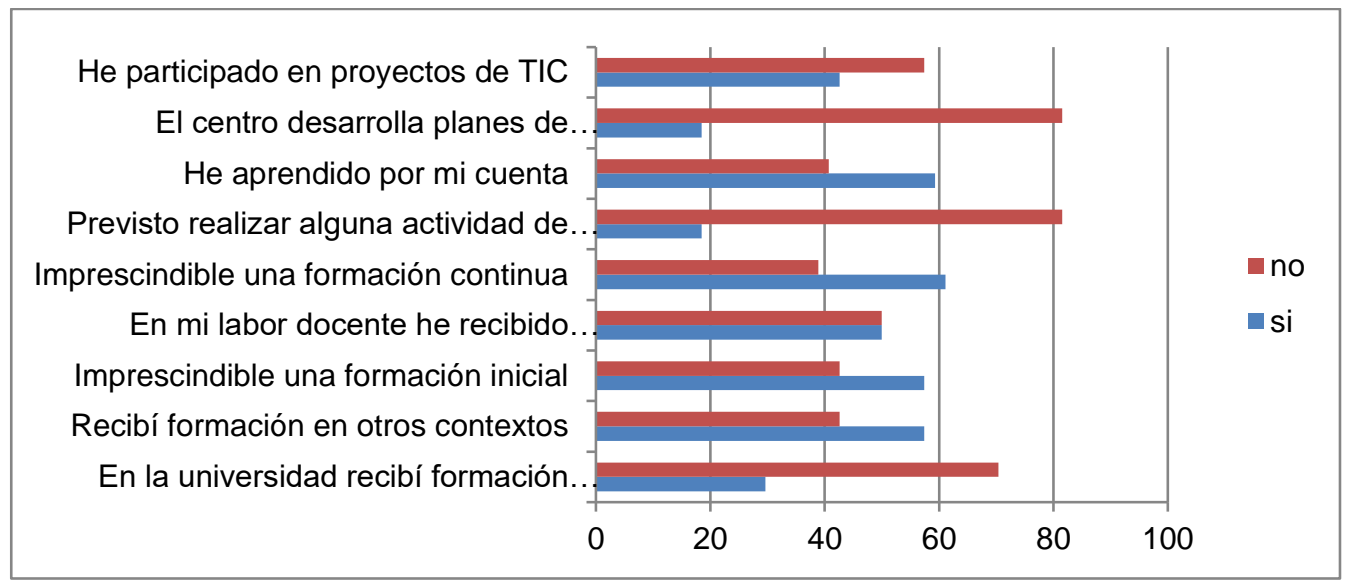

Figura 4. Formación docente

\subsection{Tecnologías y metodologías}

\subsubsection{Tecnologías emergentes}

Los resultados obtenidos muestran, como se puede apreciar en la gráfica de frecuencias, que la gran mayoría de las tecnologías emergentes que están presentes en nuestros días, nunca o rara vez han sido utilizadas por los docentes en los Colegios Rurales Agrupados de la Región de Murcia, con unos porcentajes bastante elevados. Es el caso, en primer lugar, de la impresión en 3D, la Realidad virtual y la Realidad aumentada, donde los porcentajes rondan entre el $80 \%$ y el $90 \%$, cuya frecuencia de uso es de nunca.

A continuación, a estos porcentajes tan elevados, les sigue la Robótica, la videollamada y la videoconferencia con porcentajes también altos, entre $70 \%$ y el $80 \%$ de los docentes nunca hacen uso de estas tecnologías para impartir sus clases.

En el otro extremo de la gráfica, encontramos recursos tradicionales como son los impresos, los icónicos y los sonoros, los cuales, sumando los porcentajes de las frecuencias a menudo y siempre, se encuentran entre los más utilizados por los docentes con porcentajes que rondan el $80 \%$. En especial destacar el uso de los recursos impresos, ya que el $88,9 \%$ de los docentes a menudo y siempre los utilizan.

Por otro lado, tenemos los vídeos en internet y los recursos multimedia online, que con porcentajes que se sitúan entre el $50 \%$, nos indican que son utilizados a menudo, superando los apoyados en soportes, que son utilizados alguna vez (35,2\%). Además, el libro digital, póster digital, mural digital, medios sociales, blogs y aplicaciones geolocalización, son tecnologías donde los porcentajes correspondientes están más distribuidos, pero destacamos que los más elevados suelen encontrarse entre las frecuencias rara vez y nunca. 


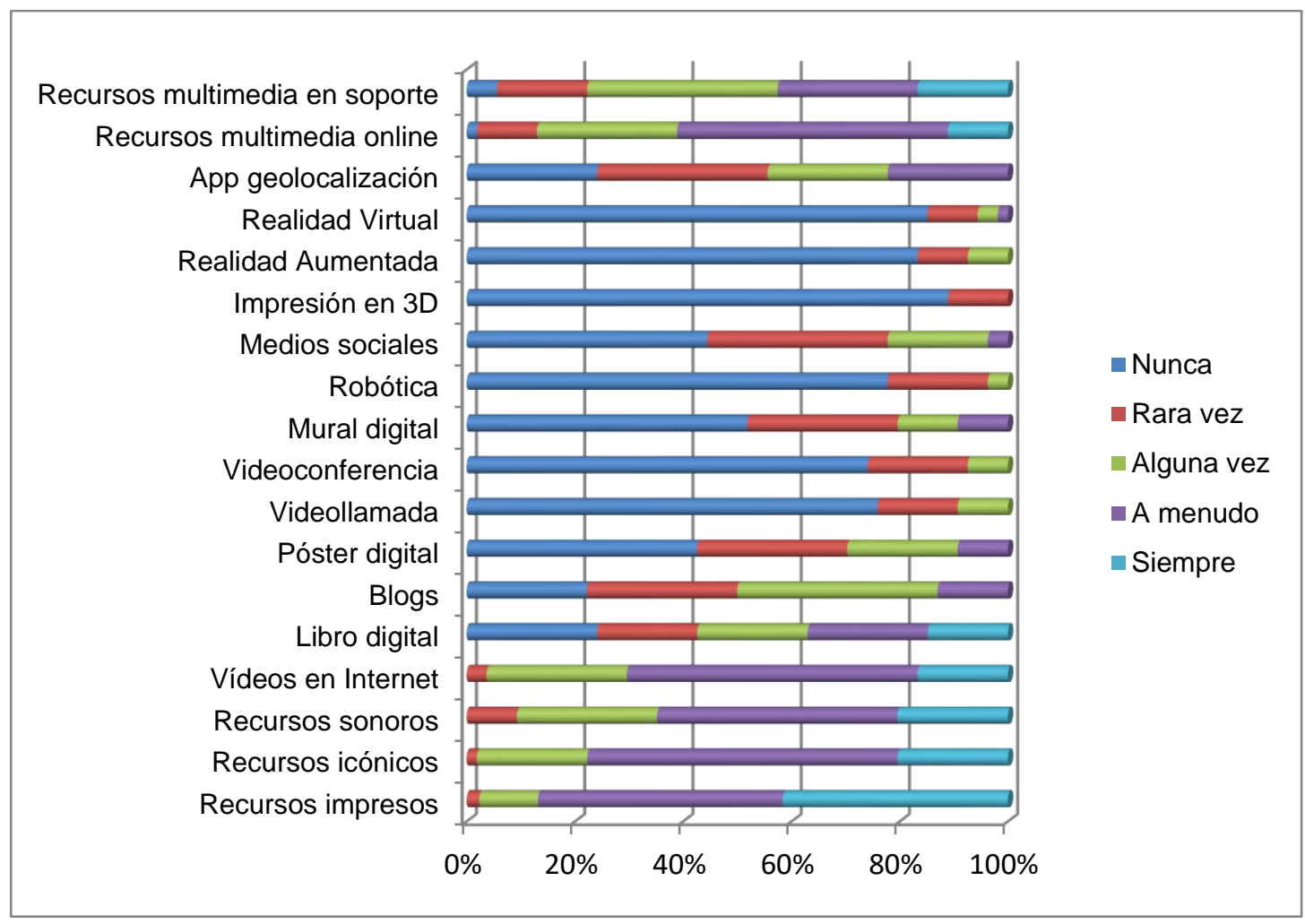

Figura 5. Frecuencia de uso de los recursos

\subsubsection{Estrategias metodológicas innovadoras}

Como bien se aprecia en la Figura 16 sobre la frecuencia de uso de estrategias metodológicas, el color que predomina es el azul más oscuro, que pertenece a no conozco, Este dato tan significativo nos dice, que menos una de las estrategias metodológicas como es el aprendizaje basado en proyectos, todas las demás son bastante desconocidas para los docentes de los colegios rurales agrupados, unas en mayor medida que otras como veremos a continuación en la Figura 17.

En cuanto a la frecuencia de uso, encontramos que la más utilizada, con diferencia, es el aprendizaje basado en proyectos, ya que si sumamos las frecuencias de a menudo y siempre nos sale un porcentaje del $33,4 \%$. Sin embargo, el resto, nunca o alguna vez han sido puestos en prácticas, por el motivo de que en gran medida son desconocidas para ellos.

En la gráfica que observamos a continuación, comprobamos que las más desconocidas para ellos son sin duda BYOD (bring your own device), y Proyectos telecolaborativos, por lo tanto los porcentajes sobre que nunca han sido utilizadas son más elevados que el resto, con un $39 \%$ y un $25 \%$. En menor medida, pero también desconocidas, tenemos la gamificación con un porcentaje de $18 \%$ y Flipped Classroom con $16 \%$. 


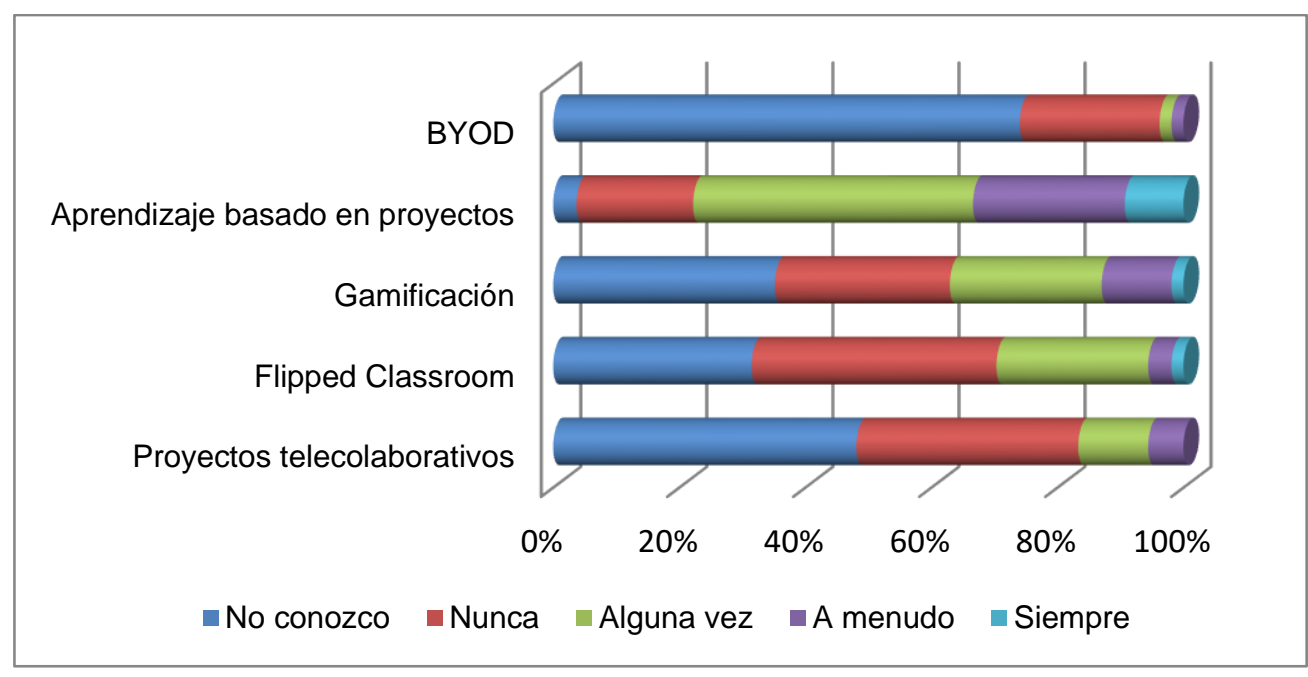

Figura 6. Frecuencia de uso de estrategias metodológicas

Además, se plantearon dos preguntas abiertas. En la primera de ellas se daba la opción de añadir otras estrategias metodológicas innovadoras que pusieran en práctica aparte de las señaladas, solamente hemos obtenido la respuesta de uno de los participantes, el cual, indica "Design thinking, aprendizaje cooperativo".

Por otra parte, la segunda cuestión abierta les pregunta si el uso de las TIC ha supuesto un cambio metodológico en sus clases, y en qué sentido se ha producido. Puesto que los 54 participantes han contestado, hemos procedido a transcribir y analizar sus respuestas mediante lo que se conoce como categorización

Se han establecido dos grandes categorías, por un lado, la información que se refiere a la metodología, y por el otro la información que nos habla sobre recursos. Una vez determinadas éstas, se ha procedido a la selección de las palabras claves de cada respuesta obtenida, constituyendo una serie de categorías, asignándole un número según la frecuencia en la que aparecen.

En primer lugar, señalar que no todos los docentes que han participado en esta pregunta han afirmado que el uso de las TIC haya supuesto un cambio metodológico en sus clases, exactamente tres de ellos comentan que "no" o "no especialmente", mientras que otro de ellos ha contestado "Ns/nc". Los 51 restantes, sí están de acuerdo con el cambio producido.

En cuanto a la categoría de metodología, existe una mayoría que apunta que sus clases son motivadoras, exactamente esta palabra aparece 26 veces a lo largo de todas las respuestas. Además, 4 veces aparece la palabra atractiva, y también aparece la palabra "amena" con una frecuencia de 3 veces, lo que quiere decir que gracias al uso de las TIC sus clases son motivadoras y atractivas y amenas.

En relación con los recursos, con una frecuencia de 5 aparece la palabra información, y con el número 3 de apariciones tenemos también las palabras rapidez e interacción, es decir, señalan que se trata de recursos que les aportan variedad de información, además de rapidez e interacción.

\section{DISCUSIÓN Y CONCLUSIONES}

Algunas de las conclusiones de nuestro trabajo coinciden con estudios realizados por otros autores, también relacionados con la escuela rural. Es el ejemplo de Del Moral, Villalustre y Neira (2014), el cual obtiene resultados que indican que el profesorado coincide en que, gracias a la integración de las TIC al currículo, se ha producido un cambio en las tareas que se desarrollan en el aula, percibiendo que los alumnos son más participativos, receptivos, y colaboradores, gracias a que las TIC posibilitan la realización de actividades dinámicas e interactivas. De este modo, la gran mayoría del profesorado opina que se han producido cambios en sus metodologías como consecuencia de la incorporación de las TIC. 
Por todo esto, también están de acuerdo, al igual que en nuestra investigación, en que es una condición necesaria la formación que reciba el profesorado, de manera que se puedan integrar las TIC con la finalidad correcta, aprovechando las posibilidades educativas que nos ofrecen.

En cuanto a la dotación tecnológica, Del Moral y Villalustre (2011), señalan que la situación de aislamiento en la que se encontraba sumida la escuela rural ha sido superada, sin embargo, no se puede olvidar los problemas que esto ha supuesto para este tipo de centros, como el retraso en la distribución del equipamiento o la incorporación pausada a las experiencias docentes, un aspecto por el que aún se sigue luchando, como hemos visto en los resultados obtenidos en el presente estudio realizado.

Respecto a los resultados obtenidos sobre la disponibilidad y uso de los recursos, se concluye que el recurso más empleado por ellos es el ordenador fijo con una diferencia significativa, mientras que en menor medida también suelen utilizar a menudo los proyectores y la Pizarra Digital Interactiva, destacando aquí los proyectores, ya que es cierto que existe un número elevado de maestros que indican no disponer de Pizarra Digital Interactiva en su aula. El motivo por el que no suelen hacer uso del resto de recursos, como tablets, ordenador portátil o smartphone, se deduce se debe a la escasa disponibilidad de éstos, ya que son los tres recursos que más faltan en las aulas según sus respuestas.

Además, podemos destacar también, que en los tiempos que corren aún quedan bastantes colegios que no disponen de una conexión a internet con una adecuada velocidad, teniendo en cuenta que casi una cuarta parte de ellos afirman tener una conexión que no es de banda ancha.

En relación con su formación docente, es significativo el dato de que la gran mayoría afirmen no haber recibido una formación inicial específica en materia de nuevas tecnologías durante sus años como universitarios, y que se hayan visto en la necesidad de formarse a través de contextos no formales, o por cuenta propia. Esto es debido a la edad media de los participantes, ya que la mayoría se encuentran entre los 35 y 50 años. Sin embargo, más de la mitad piensan que es necesaria una formación inicial, y sobre todo que es necesario actualizarse para ser profesores competentes. Pese a los datos obtenidos, es destacable señalar que existe un porcentaje muy bajo de docentes que tengan previsto realizar a corto plazo algún tipo de formación continua, o que en sus centros correspondientes se desarrollen planes de formación para el profesorado.

Como hemos señalado al introducir el tema, para que se produzca un verdadero cambio metodológico en las aulas de los CRA, no se trata exclusivamente de que los centros tengan una dotación tecnológica adecuada, si no de que los docentes sean capaces de adaptarse y ponerlas en práctica con la finalidad adecuada.

Haciendo referencia al uso de estrategias metodológicas enriquecidas con TIC, hemos analizado y conocido en primer lugar el uso, sobre las últimas tendencias en tecnologías educativas, denominando a estas como tecnologías emergentes, en comparación con recursos tradicionales. Podemos comprobar, que efectivamente, hay muy pocos maestros que hagan uso de tecnologías como: la Realidad Aumentada, la Realidad Virtual, la Robótica o las Impresiones en 3D. Sin embargo, si son más comunes entre la mayoría los recursos clásicos, sobre todo los recursos impresos e icónicos, y en menor medida recursos como videoconferencia, videollamadas, o medios sociales, llegando a la conclusión que aún les cuesta dejar de lado el papel e innovar.

Algo similar ocurre con las estrategias metodológicas que llevan a cabo. Resulta muy significativo que casi todas las que se han planteado, sean en gran medida desconocidas para ellos, o que nunca las hayan puesto en práctica, exceptuando el aprendizaje basado en proyectos. Estrategias como los Proyectos telecolaborativos, o Flipped Classroom, resultan muy innovadoras y productivas hoy en día, debido al interés y motivación que despiertan en los alumnos. Sobre este dato se llega a la conclusión, de que una mayoría del profesorado participante aún pertenece a la vieja escuela, desaprovechando todas las ventajas y oportunidades que nos brindan las TIC para el proceso de enseñanza-aprendizaje.

Algunos docentes, afirman en sus respuestas a las preguntas de carácter abierto, que no se ha producido un cambio metodológico en sus clases debido al uso de las TIC. Mientras que sí existe una gran mayoría que opina lo contrario, señalando que sus clases verdaderamente se 
han visto enriquecidas, sobre todo en lo que se refiere a la motivación, el interés que despierta en los alumnos, sus clases son más atractivas y a menas, además de la utilidad, eficacia y fácil acceso a la información que suponen los recursos.

Algunas de las conclusiones de nuestro trabajo coinciden con estudios realizados por otros autores, también relacionados con la escuela rural. Es el ejemplo de Del Moral, Villalustre y Neira (2014), el cual obtiene resultados que indican que el profesorado coincide en que gracias a la integración de las TIC al currículo, se ha producido un cambio en las tareas que se desarrollan en el aula, percibiendo que los alumnos son más participativos, receptivos, y colaboradores, gracias a que las TIC posibilitan la realización de actividades dinámicas e interactivas. De este modo, la gran mayoría del profesorado opina que se han producido cambios en sus metodologías como consecuencia de la incorporación de las TIC.

Por todo esto, también están de acuerdo, al igual que en nuestra investigación, en que es una condición necesaria la formación que reciba el profesorado, de manera que se puedan integrar las TIC con la finalidad correcta, aprovechando las posibilidades educativas que nos ofrecen.

En cuanto a la dotación tecnológica, Del Moral y Villalustre (2011), señalan que la situación de aislamiento en la que se encontraba sumida la escuela rural ha sido superada, sin embargo, no se puede olvidar los problemas que esto ha supuesto para este tipo de centros, como el retraso en la distribución del equipamiento o la incorporación pausada a las experiencias docentes, un aspecto por el que aún se sigue luchando, como hemos visto en los resultados obtenidos en el presente estudio realizado.

Respecto a los logros conseguidos con la realización del estudio, hemos podido comprobar ciertas necesidades tanto relacionadas con el uso de TIC, como con la formación que presenta el profesorado. Por este motivo, esta investigación podría servir como base de futuros proyectos de investigación mucho más profundos, que ayuden a mejorar la situación y así enriquecer la calidad de la educación en estos tipos de centros.

Una de las grandes limitaciones que se han encontrado, radicó en la dificultad para encontrar información relacionada con el tema a investigar. A lo largo de todo el proceso se ha realizado una exhaustiva búsqueda, tanto en español como en inglés, a través de la red para encontrar estudios que relacionaran el uso de las TIC con los centros en contextos rurales. Han sido escasas las publicaciones encontradas, y sobre todo han sido procedentes de la Comunidad Autónoma de Andalucía y Asturias, pero ninguna que se situara en la Región de Murcia. Otro aspecto a considerar ha sido la dificultad para obtener una muestra considerable que participara en el estudio, ya que solamente 54 docentes han colaborado. Hubiese sido ideal conseguir un número más elevado para así ser más significativo.

Como futuras líneas de investigación, podrían llevarse a cabo estudios que analizasen más ampliamente el tema consiguiendo una muestra mayor, y planteando un estudio más profundo en cuanto a dotación tecnológica se refiere, ya que este aspecto ha sido tratado de forma leve. Además sería conveniente realizar estudios cualitativos, que pudieran analizar la percepción que tiene el profesorado en cuanto a la desigualdad o diferencia que encuentran entre este tipo de centros y otros urbanos, y en el sentido que se produce. Podría ser interesante también, realizar estudios comparativos que determinaran la dotación tecnológica de unos u otros y el uso que hacen de la tecnología. Por otro lado, sería positivo incluir algunas propuestas de intervención, como diseñar e implementar estrategias didácticas y propuestas con TIC para una formación del profesorado acorde con las necesidades que nos va demandado la sociedad.

\section{ENLACES}

https://goo.gl/forms/g6jMl12lpulnrhMr1 


\section{REFERENCIAS BIBLIOGRÁFICAS}

Alcántara, M. D. (2009). Importancia de las TIC para la educación. Nuevas tecnologías. Recuperado de: https://archivos.csif.es/archivos/andalucia/ensenanza/revistas/csicsif/revista/pdf/Numero_15/ MARIA\%20DOLORES_ALCANTARA_1.pdf

Bustos, A. (2006). Los grupos multigrado de educación primaria en Andalucía (tesis doctoral). Universidad de Granada.

Bustos, A., y Román, M. (2016). La importancia de evaluar la incorporación y el uso de las TIC en educación. Revista Iberoamericana de evaluación educativa, 4(2). Recuperado de: file://C:/Users/pili_000/Downloads/4452-9414-1-PB\%20(1).pdf

Cabero, J. (2015). Reflexiones educativas sobre las tecnologías de la información y la comunicación (TIC). Tecnología, Ciencia y Educación, 1, 19-27. Recuperado de: http://hdl.handle.net/11441/32285

Corchón, E. (2005). La escuela en el medio rural: modelos organizativos. Barcelona, España: DaVinci Continental.

Cruz, T., Torres, L. M., López, E., y Ballesteros, C. (2004). Las Nuevas Tecnologías y su influencia en la mejora de las Escuelas Rurales de la comunidad Andaluza. EDUTEC 2004, 1-13. Recuperado de: http://hdl.handle.net/11441/64799

Del Moral, M. E., y Villalustre, L. (2007). Herramientas de la web 2.0 y desarrollo de proyectos colaborativos en la escuela rural. Aula Abierta, 35 (1-2). Recuperado de: http://hdl.handle.net/10651/26931

Del Moral, M. E., y Villalustre, L. (2011). Digitalización de las escuelas rurales asturianas: maestros rurales 2.0 y desarrollo local. Profesorado. Revista de Currículum y Formación de Profesorado, 15(2), 109-123. Recuperado de: http://www.redalyc.org/pdf/567/56719129008.pdf

Del Moral, M. E., Villalustre L., y Neira, M. D. R. (2014). Oportunidades de las TIC para la innovación educativa en las escuelas rurales de Asturias. Aula abierta, 42(1), 61-67. doi: https://doi.org/10.1016/S0210-2773(14)70010-1

Del Moral, M. E., Villalustre, L., y Neira, M. D. R. (2014). Variables asociadas a la cultura innovadora con TIC en escuelas rurales. Profesorado. Revista de Currículum y Formación de Profesorado, 18(3), 9-25. Recuperado de: http://www.redalyc.org/pdf/567/56733846002.pdf

Hinojo, F. J., Raso, F., y Hinojo, M. A. (2010). Análisis de la organización de la escuela rural en Andalucía: problemática y propuestas para un desarrollo de calidad. REICE. Revista Iberoamericana Sobre Calidad, Eficacia y Cambio en Educación, 8(1). Recuperado de: http://www.redalyc.org/html/551/55113489006/

INTEF (2017). Marco Común de Competencia Digital Docente. Recuperado de: http://educalab.es/documents/10180/12809/Marco+competencia+digital+docente+2017/afb0 7987-1ad6-4b2d-bdc8-58e9faeeccea

McMillan, J. H. y Schumacher, S. (2005). Investigación educativa una introducción conceptual. Madrid, España: Pearson educación.

Moscoso, J. N. (2017). Los métodos mixtos en la investigación en educación: hacia un uso reflexivo. Cadernos de Pesquisa, 47(164), 632-649. doi: http://dx.doi.org/10.1590/198053143763 
Raso, F. (2012). La escuela rural Andaluza y su profesorado ante las tecnologías de la información y la comunicación (TICs): Estudio Evaluativo (tesis doctoral). Universidad de Granada.

Raso, F., Hinojo, M. A., y Solá, J. M. (2015). Integración y uso docente de las tecnologías de la información y la comunicación (TIC) en la escuela rural de la provincia de Granada: estudio descriptivo. REICE. Revista Iberoamericana sobre Calidad, Eficacia y Cambio en Educación, 13(1), 139-159. Recuperado de: https://revistas.uam.es/index.php/reice/article/view/2803/3018

Recio, M. S. (2016). Formación en TIC del profesorado de Educación Infantil: uso de las tecnologías y cambio metodológico (tesis doctoral). Universidad de Murcia.

Vuorikari, R., Punie, Y., Carretero, S., y Van Den Brande, G. (2016). DigComp 2.0: The digital competence framework for citizens. Update phase 1: The conceptual reference model. doi: $10.2791 / 607218$

\section{INFORMACIÓN SOBRE LOS AUTORES}

Ma Pilar Segura García

Universidad de Murcia

Estudiante del Máster de Tecnología Educativa

\section{Isabel Ma Solano Fernádez}

Universidad de Murcia

Profesora del Departamento de Didática y Organización Escolar. Miembro del Grupo de Investigación de Tecnología Educativa.

\section{Ma del Sánchez Vera}

Universidad de Murcia

Profesora del Departamento de Didática y Organización Escolar. Miembro del Grupo de Investigación de Tecnología Educativa.

Los textos publicados en esta revista están sujetos a una licencia de Reconocimiento 4.0 España de Creative Commons. Puede copiarlos, distribuirlos, comunicarlos públicamente y hacer obras derivadas siempre que reconozca los créditos de las obras (autoría, nombre de la revista, institución editora) de la manera especificada por los autores o por la revista. La licencia completa se puede consultar en:Licencia Creative Commons Atribución-NoComercial-Compartir por igual 4.0 Internacional. 